\title{
Regularization of $p$-Adic Distributions Associated to Functions on $p$-Adic Fields With Moderate Variation
}

\author{
Altan Erdoğan
}

\begin{abstract}
The $p$-adic distributions attached to ordinary functions defined on $p$-adic fields with moderate variation are studied. We first give a sufficient growth condition on ordinary functions to construct $p$-adic distributions. Then a moderate variation condition on functions for regularization of these $p$-adic distributions is imposed which provides a general method to construct $p$-adic measures. The $p$-adic integrals against these measures are also explicitly transformed to integrals against Bernoulli measures.
\end{abstract}

Keywords: $p$-Adic analysis; $p$-Adic measures; $p$-Adic integration, Bernoulli measures, $p$-Adic gamma measures AMS Subject Classification (2020): Primary: $11 S 80$

\section{Introduction}

The theory $p$-adic integration has been an important tool in number theory for many years. The construction of the $p$-adic zeta function by $p$-adic interpolation of the complex Riemann zeta function $\zeta(s)$ at negative integers was achieved by Leopoldt and Kubota by using the ( $p$-adic) limits

$$
\lim _{M \rightarrow \infty} \frac{1}{p^{M}} \sum_{j=0}^{p^{M}-1} f(x+j)
$$

for a locally analytic function $f$ on $\mathbb{Z}_{p}$ [1]. This idea has been conceptualized by expressing $\zeta(-r)$ for $r \in \mathbb{Z}_{\geq 1}$ as the $p$-adic integral of $x^{r}$ against the $p$-adic measure $\mu_{\alpha, 1}$ called as Mazur's Bernoulli measure [9]. Indeed more generally one can define a $p$-adic $L$-function as the $p$-adic integral of a Dirichlet character against a $p$-adic measure. Today it is well known that the special values of complex $L$-functions are also expressed in terms of $p$-adic integrals and this relation is also reflected to the $p$-adic properties of modular forms and moduli spaces $[7,8]$.

In a simple setting, a $p$-adic distribution denoted by $\mu$ on a compact subset $X$ of $\mathbb{Q}_{p}$ can be defined as a finitely additive map from the compact open subsets of $X$ to $\mathbb{C}_{p}$. Explicitly if $U \subset X$ is equal to the disjoint union of compact open subsets $U_{1}, \ldots, U_{n}$ then $\mu(U)=\mu\left(U_{1}\right)+\ldots+\mu\left(U_{1}\right)$. Indeed it is enough to check the additivity condition on the 
compact open subsets of the form $a+\left(p^{N}\right)$ contained in $X$, i.e. $\mu$ extends to a $p$-adic distribution on $X$ if and only if

$$
\mu\left(a+\left(p^{N}\right)\right)=\sum_{b=0}^{p-1} \mu\left(a+b p^{N}+\left(p^{N+1}\right)\right)
$$

for any $a+\left(p^{N}\right)$ contained in $X$. We will call (1.1) as the distribution property. If additionally $\mu$ is bounded then we call $\mu$ as a $p$-adic measure on $X$. The reader is referred to $[3,6,9]$ for the properties as well as equivalent characterizations of $p$-adic distributions and measures. We will mainly take $X$ to be $\mathbb{Z}_{p}$ or $\mathbb{Z}_{p}^{*}$.

The $p$-adic integral of a continuous function $f(x)$ on $X$ against a $p$-adic measure $\mu$ denoted by $\int_{X} f \mu$ is defined as

$$
\int_{X} f \mu=\lim _{N \rightarrow \infty} \sum_{U \subset X} f(\tilde{a}) \mu(U)
$$

where $U$ ranges over the compact open subsets of $X$ of the form $a+\left(p^{N}\right)$ and $\tilde{a} \in U$ is arbitrary. Note that this limit exists and is independent of the choice of $\tilde{a} \in U[9$, Theorem 6 of $\S I I]$.

The standard examples of $p$-adic measures are Bernoulli measures, $p$-adic Gamma measures and the measure $\mu_{z}$ defined as

$$
\mu_{z}\left(a+\left(p^{N}\right)=z^{a} /\left(1-z^{p^{N}}\right)\right.
$$

where $z \in \mathbb{C}_{p}$ with $|z-1|_{p} \geq 1$ (See [9], [5] and [10] respectively). Bernoulli measures is core for construction of $p$-adic zeta functions. The $p$-adic Gamma measures give a $p$-adic analogue of Euler constant [4]. Also the measure $\mu_{z}$ has an important role in the theory of $p$-adic polylogarithms [2].

We briefly recall the Bernoulli measures referring to [9] for the details. The $k$-th Bernoulli distribution $\mu_{B, k}$ is defined as

$$
\mu_{B, k}\left(a+\left(p^{N}\right)\right)=p^{N(k-1)} B_{k}\left(a / p^{N}\right)
$$

where $B_{k}(x)$ is the $k$-the Bernoulli polynomial. Note that $\mu_{B, k}$ is unbounded, so is not a measure. The $k$-th Bernoulli measure is obtained by regularization of $\mu_{B, k}$ as follows. Let $\alpha \in \mathbb{Z}_{\geq 1}$ for which $(p, \alpha)=1$. Then it is clear that for any distribution $\mu$ on $\mathbb{Z}_{p}$, the map $U \mapsto \mu(\alpha U)$ on the compact open subsets of $\mathbb{Z}_{p}$ is also a distribution and so is

$$
U \mapsto \mu_{k, \alpha}(U):=\mu_{k, \alpha}(U)-\alpha^{-k} \mu_{k, \alpha}(\alpha U) .
$$

It turns out that $\mu_{k, \alpha}$ is bounded, indeed $\left|\mu_{k, \alpha}(U)\right|_{p} \leq 1$ and so is a $p$-adic measure on $\mathbb{Z}_{p}$. The $p$-adic Gamma measures are also constructed in a similar way [4].

In this paper we will generalize this method of construction of $p$-adic distributions and measures on $\mathbb{Z}_{p}^{*}$ and show that we can associate $p$-adic measures to ordinary functions satisfying some growth and variation conditions. In particular we will see that the Bernoulli measures and $p$-adic Gamma measures correspond to polynomials and $x \log _{p}(x)-x$ respectively where $\log _{p}$ is Iwasawa's $p$-adic logarithm, but the method provided here is also valid for a larger class of functions. We will also give an expression for $p$-adic integrals against these measures in terms of Mazur's Bernoulli measure.

We fix the following notation for the rest of the paper. We will denote the ring of $p$-adic integers and its field of fractions by $\mathbb{Z}_{p}$ and $\mathbb{Q}_{p}$ respectively. Also $\mathbb{C}_{p}$ denotes the completion of a fixed algebraic closure of $\mathbb{Q}_{p}$ endowed with the normalized $p$-adic norm as $|p|_{p}=1 / p$. The group of units of $\mathbb{Z}_{p}$ is denoted by $\mathbb{Z}_{p}^{*}$, i.e. $\mathbb{Z}_{p}^{*}=\left\{\alpha \in \mathbb{Z}_{p}:|\alpha|=1\right\}$.

\section{Admissible functions with moderate variation}

We give a basic definition for the functions that we will work on. Let $R \geq 0$ be a real number and $D_{R}:=\{x \in$ $\left.\left.\mathbb{Q}_{p}|| x\right|_{p} \geq R, x \notin \mathbb{Z}_{\leq 0}\right\}$.

Definition 2.1. We say that a function $f$ defined on $D_{R}$ is admissible if there exists $k \in \mathbb{Z}_{\geq 1}$ for which the following are satisfied;

i) The pointwise limit $F(x):=\lim _{M \rightarrow \infty} p^{M(k-1)} \sum_{j=0}^{p^{M}-1} f\left(\frac{x+j}{p^{M}}\right)$ exists for any $x \in \mathbb{Q}_{p}$ with $x \notin \mathbb{Z}_{\leq 0}$

ii) For some $\alpha \in \mathbb{Z}_{\geq 2}$ with $(p, \alpha)=1$ the pointwise limit $F_{\alpha}(x):=\lim _{M \rightarrow \infty} p^{M(k-1)} \sum_{j=0}^{p^{M}-1} f\left(\frac{x+j}{\alpha p^{M}}\right)$ exists for any $x \in \mathbb{Q}_{p}$ with $x \notin \mathbb{Z}_{\leq 0}$. 
For example $f(x)=x^{k}$ and $f(x)=x^{k} \log _{p}(x)$ for $k \in \mathbb{Z}_{\geq 0}$ are admissible functions, and $\alpha \geq 2$ can be any integer with $(p, \alpha)=1$. In the sequel we always consider an admissible function together with a fixed $\alpha$ satisfying condition ii) of Definition 2.1 without any further reference. Now we show that admissible functions induce $p$-adic distributions.

Proposition 2.1. Let $f$ be an admissible function on some $D_{R}$. Then the maps $\mu_{f}$ and $\mu_{f, \alpha}$ defined as

$$
\begin{aligned}
& \mu_{f}\left(a+\left(p^{N}\right)\right)=p^{N(k-1)} F\left(a / p^{N}\right) \\
& \mu_{f, \alpha}\left(a+\left(p^{N}\right)\right)=p^{N(k-1)} F_{\alpha}\left(a / p^{N}\right)
\end{aligned}
$$

on the compact open subsets of $\mathbb{Z}_{p}^{*}$ extend to $p$-adic distributions on $\mathbb{Z}_{p}^{*}$.

Proof. We need to check the distribution property (1.1). Now we have that

$$
\begin{aligned}
& \sum_{b=0}^{p-1} \mu_{f, \alpha}\left(a+b p^{N}+\left(p^{N+1}\right)\right)=\sum_{b=0}^{p-1} p^{(N+1)(k-1)} F_{\alpha}\left(\frac{a+b p^{N}}{p^{N+1}}\right) \\
& =p^{(N+1)(k-1)} \lim _{M \rightarrow \infty} p^{M(k-1)} \sum_{b=0}^{p-1} \sum_{j=0}^{p^{M}-1} f\left(\frac{\left(a+b p^{N}\right) / p^{N+1}+j}{\alpha p^{M}}\right) \\
& =p^{N(k-1)} \lim _{M \rightarrow \infty} p^{(M+1)(k-1)} \sum_{b=0}^{p-1} \sum_{j=0}^{p^{M}-1} f\left(\frac{\left(a / p^{N}\right)+(b+j p)}{\alpha p^{M+1}}\right) \\
& =p^{N(k-1)} \lim _{M^{\prime} \rightarrow \infty} p^{M^{\prime}(k-1)} \sum_{j^{\prime}=0}^{p^{M^{\prime}}-1} f\left(\frac{\left(a / p^{N}\right)+j^{\prime}}{\alpha p^{M^{\prime}}}\right)
\end{aligned}
$$

where $M^{\prime}=M+1$ and $j^{\prime}=b+j p$. But by definition the last sum is equal to

$$
p^{N(k-1)} F_{\alpha}\left(a / p^{N}\right)=\mu_{f, \alpha}\left(a+\left(p^{N}\right)\right)
$$

which completes the proof (Note that the above arguments are also valid for $\alpha=1$.)

Remark 2.1. The condition $x \notin \mathbb{Z}_{\leq 0}$ is redundant in some cases. For example if $f(x)$ is a polynomial then $f$ is well defined on $\mathbb{Q}_{p}$ and so $\mu_{f}$ and $\mu_{f, \alpha}$ extend to $p$-adic distributions also on $\mathbb{Z}_{p}$. But for example if $f(x)=\log _{p}(x)$ which is not defined at $x=0$, then the condition $x \notin \mathbb{Z}_{\leq 0}$ is necessary.

Now we conceptualize the notion of regularization of $p$-adic distributions obtained in Proposition 2.1. We adopt the notation of Definition 2.1 and Proposition 2.1. Recall that since $\mu_{f, \alpha}$ is a $p$-adic distribution, the map

$$
U \mapsto \mu_{f, \alpha}(\alpha U)
$$

also extends to a $p$-adic distribution. Explicitly we have that

$$
\mu_{f, \alpha}(\alpha U)=p^{N(k-1)} F_{\alpha}\left(\left\{\alpha a / p^{N}\right\}\right)
$$

where $\{x\}$ denotes the fractional part of $x$.

Definition 2.2. Let $f$ be an admissible function and $\mu_{f}^{(\alpha)}$ be the $p$-adic distribution defined as

$$
\mu_{f}^{(\alpha)}(U)=\mu_{f}(U)-\mu_{f, \alpha}(\alpha U)=p^{N(k-1)} F\left(a / p^{N}\right)-p^{N(k-1)} F_{\alpha}\left(\left\{\alpha a / p^{N}\right\}\right)
$$

for any compact open subset $U$ of $\mathbb{Z}_{p}^{*}$. If $\mu_{f}^{(\alpha)}$ is bounded, and so extends to a $p$-adic measure then we say that $\mu_{f}^{(\alpha)}$ is a regularization of $\mu_{f, 1}$.

Now we will give two main results. The first theorem below presents a sufficient condition on $f$ which guarantees the boundedness of $\mu_{f}^{(\alpha)}$. The importance of it is that a moderate variation for an admissible function $f$ is enough for the boundedness of $\mu_{f}^{(\alpha)}$. The second theorem will be useful to express the $p$-adic integrals against $\mu_{f}^{(\alpha)}$ in terms of $p$-adic integrals against Mazur's Bernoulli measure. 
Theorem 2.1. Let $f$ be an admissible function, and $k \in \mathbb{Z}_{\geq 1}$. Suppose that

$$
\sup _{u \in \mathbb{Z}_{p}}\left\{|f(y+u)-f(y)|_{p}\right\}=O\left(\left|y^{k-1}\right|_{p}\right) \text { as }|y|_{p} \rightarrow \infty
$$

Then $\left|\mu_{f}^{(\alpha)}\right|_{p}$ is bounded, and so is a regularization of $\mu_{f, 1}$.

Proof. By definition we have

$$
\mu_{f}^{(\alpha)}\left(a+\left(p^{N}\right)\right)=p^{N(k-1)} \lim _{M \rightarrow \infty} p^{M(k-1)} \sum_{j=0}^{p^{M}-1}\left[f\left(\frac{\left(a / p^{N}\right)+j}{p^{M}}\right)-f\left(\frac{\left\{\alpha a / p^{N}\right\}+j}{\alpha p^{M}}\right)\right] .
$$

Put $b=a / p^{N}$. So

$$
\frac{\{\alpha b\}+j}{\alpha p^{M}}=\frac{\alpha b-[\alpha b]+j}{\alpha p^{M}}=\frac{b+\left(\frac{j-[\alpha b]}{\alpha}\right)}{p^{M}}
$$

Since $p \nmid \alpha$, for a fixed $M$ and any $j \in\left\{0,1, \ldots, p^{M}-1\right\}$, there exists a unique $j^{\prime} \in\left\{0,1, \ldots, p^{M}-1\right\}$ such that $j^{\prime} \equiv \alpha j+[\alpha b]\left(\bmod p^{M}\right)$, so the mapping $j \mapsto j^{\prime}$ on the set $\left\{0,1,2, \ldots, p^{M}-1\right\}$ is bijective. But then

$$
\frac{b+j}{p^{M}}-\frac{\{\alpha b\}+j^{\prime}}{\alpha p^{M}}=\frac{b+j}{p^{M}}-\frac{b+\left(\frac{j^{\prime}-[\alpha b]}{\alpha}\right)}{p^{M}}=\frac{j-\left(\frac{j^{\prime}-[\alpha b]}{\alpha}\right)}{p^{M}} \in \mathbb{Z}_{p} .
$$

So by hypothesis for large enough $M$ we have

$$
\left|f\left(\frac{b+j}{p^{M}}\right)-f\left(\frac{\{\alpha b\}+j^{\prime}}{\alpha p^{M}}\right)\right|_{p} \leq K\left|\frac{b+j}{p^{M}}\right|_{p}^{k-1}=K \cdot p^{M(k-1)}|b+j|_{p}^{k-1}
$$

for some $K>0$. Now we distinguish two cases. First suppose that $N=0$, so $b=a \in \mathbb{Z}_{p}$. But then since $b+j \in \mathbb{Z}_{p}$ we have that

$$
K \cdot p^{M(k-1)}|b+j|_{p}^{k-1} \leq K \cdot p^{M(k-1)} \Longrightarrow\left|\mu\left(\mathbb{Z}_{p}^{*}\right)\right|_{p} \leq \lim _{M \rightarrow \infty}\left|p^{M(k-1)}\right|_{p} K \cdot p^{M(k-1)}=K .
$$

Now suppose that $N \geq 1$. Since $(a, p)=1$, we have $|b|_{p}>1$. But in this case $|b+j|_{p}=|b|_{p}$ and so

$$
\left|f\left(\frac{b+j}{p^{M}}\right)-f\left(\frac{\{\alpha b\}+j^{\prime}}{\alpha p^{M}}\right)\right|_{p} \leq K p^{M(k-1)}|b|_{p}^{k-1}=K p^{M(k-1)} p^{N(k-1)}
$$

which implies that $\left|\mu_{f}^{(\alpha)}\left(a+\left(p^{N}\right)\right)\right|_{p} \leq K$ as desired.

Theorem 2.2. Let $f$ be a function satisfying hypothesis of Theorem 2.1, and $M \in \mathbb{Z}_{\geq 1}$. Suppose that there exist $k \in \mathbb{Z}$ and functions $h(y)$ and $T(y, u, v)$ depending on $f$ such that for all $y, u, v \in \mathbb{Q}_{p}$ with $|y|_{p} \geq p^{M},|u-y|_{p}<p^{M},|v-y|_{p}<p^{M}$ and $u-v \in \mathbb{Z}_{p}$ the following are satisfied;

i) $f(y+u)-f(y+v)=(u-v) h(y)+T(y, u, v)$,

ii) The pointwise limit $H(a):=\lim _{r \rightarrow \infty} p^{r(k-1)} h\left(a / p^{r}\right)$ exists and is bounded on $\mathbb{Z}_{p}^{*}$ and

iii) $|T(y, v, u)|_{p}=O\left(\left|\frac{y^{k-2}}{p^{M}}\right|_{p}\right)$ as $|y|_{p} \rightarrow \infty$.

Then there exists $K>0$ such that for all $a$ and $N$,

$$
\left|\mu_{f}^{(\alpha)}\left(a+\left(p^{N}\right)\right)-H(a) \mu_{\alpha, 1}\left(a+\left(p^{N}\right)\right)\right|_{p} \leq K / p^{N}
$$


Proof. We adopt the same notation of the proof of Theorem 2.1. We set $y=\left(a / p^{N}\right) / p^{M}, u=j / p^{M}$ and $v=$ $\left(j^{\prime}-[\alpha b]\right) /\left(\alpha p^{M}\right)$. So the hypothesis on $y, u$ and $v$ are clearly satisfied. Then

$$
\begin{aligned}
\mu_{f}^{(\alpha)}\left(a+\left(p^{N}\right)\right) & =p^{N(k-1)} \lim _{M \rightarrow \infty} p^{M(k-1)} \sum_{j=0}^{p^{M}-1}\left[f\left(\frac{\left(a / p^{N}\right)+j}{p^{M}}\right)-f\left(\frac{\left\{\alpha a / p^{N}\right\}+j}{\alpha p^{M}}\right)\right] \\
& =\lim _{M \rightarrow \infty} p^{(M+N)(k-1)} h\left(a / p^{M+N}\right) \sum_{j=0}^{p^{M}-1} \frac{j-\left(j^{\prime}-\left[\alpha a / p^{N}\right]\right) / \alpha}{p^{M}}+p^{N(k-1)} \lim _{M \rightarrow \infty} p^{M(k-1)} \sum_{j=0}^{p^{M}-1} T(y, u, v) \\
& =H(a) \mu_{\alpha, 1}\left(a+\left(p^{N}\right)\right)+p^{N(k-1)} \lim _{M \rightarrow \infty} p^{M(k-1)} \sum_{j=0}^{p^{M}-1} T(y, u, v) .
\end{aligned}
$$

Now we use the hypothesis iii) and have that

$$
\begin{aligned}
\mid \mu_{f}^{(\alpha)}\left(a+\left(p^{N}\right)\right) & -\left.H(a) \mu_{\alpha, 1}\left(a+\left(p^{N}\right)\right)\right|_{p}=\left|p^{N(k-1)} \lim _{M \rightarrow \infty} p^{M(k-1)} \sum_{j=0}^{p^{M}-1} T(y, u, v)\right|_{p} \\
& \leq \frac{1}{p^{N(k-1)}} \lim _{M \rightarrow \infty} \frac{1}{p^{M(k-1)}} p^{M} K\left|a^{k-2}\right|_{p} p^{(M+N)(k-2)} \\
& =K / p^{N}
\end{aligned}
$$

as desired.

An immediate consequence of Theorem 2.2 is the relation between integrals against $\mu_{f}^{(\alpha)}$ and Mazur's measure $\mu_{\alpha, 1}$.

Corollary 2.1. Let $f$ be given as in Theorem 2.2 and suppose that the corresponding function $H(x)$ defined in Theorem 2.2 is integrable against $\mu_{\alpha, 1}$. Let $g(x)$ be a continuous function on $\mathbb{Z}_{p}^{*}$. Then

$$
\int_{\mathbb{Z}_{p}^{*}} g(x) d \mu_{f}^{(\alpha)}=\int_{\mathbb{Z}_{p}^{*}} g(x) H(x) d \mu_{\alpha, 1}
$$

Proof. By definition

$$
\begin{aligned}
& \int_{\mathbb{Z}_{p}^{*}} g(x) d \mu_{f}^{(\alpha)}=\lim _{N \rightarrow \infty} S_{N}, \text { where } S_{N}=\sum_{a=0}^{p^{N}-1} g(a) \mu_{f}^{(\alpha)}\left(a+\left(p^{N}\right)\right) \\
& \int_{\mathbb{Z}_{p}^{*}} g(x) H(x) d \mu_{\alpha, 1}=T_{N}, \text { where } T_{N}=\sum_{a=0}^{p^{N}-1} g(a) H(a) \mu_{\alpha, 1}\left(a+\left(p^{N}\right)\right)
\end{aligned}
$$

Now we see that

$$
\begin{aligned}
& \left|S_{N}-T_{N}\right|_{p}=\left|\sum_{a=0}^{p^{N}-1} g(a)\left[\mu_{f}^{(\alpha)}\left(a+\left(p^{N}\right)\right)-H(a) \mu_{\alpha, 1}\left(a+\left(p^{N}\right)\right)\right]\right|_{p} \\
& \leq \max _{0 \leq a \leq p^{N}-1}\left|g(a)\left[\mu_{f}^{(\alpha)}\left(a+\left(p^{N}\right)\right)-H(a) \mu_{\alpha, 1}\left(a+\left(p^{N}\right)\right)\right]\right|_{p}
\end{aligned}
$$

But since $g(x)$ is continuous on $\mathbb{Z}_{p}$ it is also bounded, say $g(x) \leq M$. So by Theorem 2.2 there exists a constant $K$ such that

$$
\left|S_{N}-T_{N}\right|_{p} \leq \frac{K M}{p^{N}} \Longrightarrow\left|S_{N}-T_{N}\right|_{p} \rightarrow 0 \text { as } N \rightarrow \infty
$$




\section{Examples}

We devote this section to examples. We may omit some calculations for which many of them follow by considering the power series expansion of $\log _{p}(x)$.

Example 3.1. Let $f(x)=x^{k}$ (or any monic polynomial of degree $k$ ). Then

$$
\begin{aligned}
F_{\alpha}(x) & =\lim _{M \rightarrow \infty} p^{M(k-1)} \sum_{j=0}^{p^{M}-1}\left(\frac{x+j}{\alpha p^{M}}\right)^{k}=\alpha^{-k} \lim _{M \rightarrow \infty} \frac{1}{p^{M}} \sum_{j=0}^{p^{M}-1}(x+j)^{k} \\
& =\alpha^{-k} \lim _{M \rightarrow \infty} \frac{1}{p^{M}} \sum_{j=0}^{p^{M}-1} \sum_{l=0}^{k}\left(\begin{array}{l}
k \\
l
\end{array}\right) x^{k-l} j^{l}=\alpha^{-k} \lim _{M \rightarrow \infty} \frac{1}{p^{M}} \sum_{l=0}^{k}\left(\begin{array}{c}
k \\
l
\end{array}\right) x^{k-l} \frac{B_{l+1}\left(p^{M}\right)-B_{l+1}(0)}{l+1} \\
& =\alpha^{-k} \sum_{l=0}^{k}\left(\begin{array}{l}
k \\
l
\end{array}\right) x^{k-l} \frac{1}{l+1} \lim _{M \rightarrow \infty} \frac{B_{l+1}\left(p^{M}\right)-B_{l+1}(0)}{p^{M}}=\left.\alpha^{-k} \sum_{l=0}^{k}\left(\begin{array}{l}
k \\
l
\end{array}\right) x^{k-l} \frac{1}{l+1} \frac{d B_{l+1}(x)}{d x}\right|_{x=0} \\
& =\alpha^{-k} \sum_{l=0}^{k}\left(\begin{array}{l}
k \\
l
\end{array}\right) x^{k-l} B_{l}(0)=\alpha^{-k} B_{k}(x)
\end{aligned}
$$

So we have that

$$
\mu_{f}^{(\alpha)}\left(a+\left(p^{N}\right)\right)=p^{N(k-1)}\left[B_{k}\left(a / p^{N}\right)-\alpha^{-k} B_{k}\left(\left\{\alpha a / p^{N}\right\}\right)\right] .
$$

Note that by Proposition $2.1, \mu_{f}^{(\alpha)}$ is a $p$-adic distribution. We also see that

$$
f(y+u)-f(y)=k u y^{k-1}+O\left(y^{k-2}\right) .
$$

So for any $u \in \mathbb{Z}_{p}$ and $|y|_{p}>1$, we obtain

$$
|f(y+u)-f(y)|_{p} \leq\left|y^{k-1}\right|_{p} .
$$

Hence $f(x)$ satisfies the hypothesis of Theorem 2.1 and so $\mu_{f}^{(\alpha)}$ is a $p$-adic measure. Indeed $\mu_{f}^{(\alpha)}$ is the $k$-th Bernoulli measure. We may go further and use Theorem 2.2. We compute that

$$
h(y)=k y^{k-1}, T(y, u, k)=y^{k-2}+(\text { terms with lower degree in } y)
$$

so that $f(x)$ satisfies the hypothesis of Theorem 2.2. Also $H(x)=k x^{k-1}$, so by Corollary 2.1 we have that

$$
\int_{\mathbb{Z}_{p}^{*}} g(x) d \mu_{\alpha, k}=\int_{\mathbb{Z}_{p}^{*}} k g(x) x^{k-1} d \mu_{\alpha, 1} .
$$

Example 3.2. Let $f(x)=\log _{p}(x)$. Recall that $\log _{p}(p)=0$. It is again easy to check that $f(x)=\log _{p}(x)$ satisfies the hypothesis of Theorem 2.1 with $k=0$;

$$
\begin{aligned}
f(y+u)-f(y) & =\log _{p}(y+u)-\log _{p}(y)=\log _{p}\left(1+\frac{u}{y}\right) \\
& \Longrightarrow|f(y+u)-f(y)|_{p}=|u / y|_{p} \leq\left|y^{-1}\right|_{p}
\end{aligned}
$$

Now for the corresponding $p$-adic distribution $\mu_{f, \alpha}$ we have that

$$
\begin{aligned}
\mu_{f, \alpha}(\alpha U) & =\frac{1}{p^{N}} \lim _{M \rightarrow \infty} \frac{1}{p^{M}} \sum_{j=0}^{p^{M}-1} \log _{p}\left(\frac{\left\{\alpha a / p^{N}\right\}+j}{\alpha p^{M}}\right) \\
& =\frac{1}{p^{N}} \lim _{M \rightarrow \infty} \sum_{j=0}^{p^{M}-1} \frac{1}{p^{M}}\left(\log _{p}\left(\frac{\left\{\alpha a / p^{N}\right\}+j}{p^{M}}\right)-\log _{p}(\alpha)\right) \\
& =\mu_{f, 1}(\alpha U)-\frac{\log _{p}(\alpha)}{p^{N}}
\end{aligned}
$$


So we obtain that

$$
\begin{aligned}
\mu_{f}^{(\alpha)}(U) & =\mu_{f, 1}(U)-\mu_{f, \alpha}(\alpha U) \\
& =\mu_{f, 1}(U)-\mu_{f, 1}(\alpha U)+\frac{\log _{p}(\alpha)}{p^{N}} .
\end{aligned}
$$

But this is exactly the same measure denoted by $\nu_{1, \alpha}$ which is the regularization of $\nu_{G, 1}$ in the notation of [4]. Also we have that $\left|\mu_{f}^{(\alpha)}(U)\right|_{p} \leq 1$ which follows as $K=1$ in the notation of Theorem 2.1. Also in the notation of Theorem 2.2 we see that $H(x)=1 / x$.

Example 3.3. Now let $f(x)=x \log _{p} x-x$. Then

$$
\begin{aligned}
f(y+u)-f(y) & =(y+u) \log _{p}(y+u)-(y+u)-y \log _{p}(y)+y \\
& =y \log _{p}(1+u / y)+u\left(\log _{p}(y+u)-1\right)
\end{aligned}
$$

The first term is bounded by 1 . Now let $y=1 /\left(v p^{r}\right)$ where $|v|_{p}=1$ and $r \in \mathbb{Z}_{\geq 1}$. Then $\log _{p}(y+u)=\log _{p}\left(1+u v p^{r}\right)$, and so the second term is also bounded by 1 . Hence we have $k=1$ and $K=1$ in Theorem 2.1. Then it follows that

$$
\begin{aligned}
\mu_{f}^{(\alpha)}(U) & =\mu_{f, 1}(U)-\mu_{f, \alpha}(\alpha U) \\
& =\mu_{f, 1}(U)-\frac{1}{\alpha} \mu_{f, 1}(\alpha U)+\frac{\log _{p}(\alpha)}{\alpha}\left(\left\{\frac{\alpha a}{p^{N}}\right\}-\frac{1}{2}\right) .
\end{aligned}
$$

In this case we obtain the $p$-adic Gamma measure $v_{0, \alpha}$ of J. Diamond [4]. Also if we carry on the calculations of Theorem 2.2 with $k=1$ we see that $h(x)=H(x)=\log _{p}(x)$.

\section{References}

[1] Kubota, T., Leopold H.W.; Eine p-adische Theorie der Zetawerte, I. Journ. Reine Angew. Math., 214/215, 328-339, (1964).

[2] Coleman, R.F.: Dilogarithms, Regulators and p-adic L-functions, Invent. Math.,69, 171-208, (1982).

[3] Washington, L.C.: Introduction to Cyclotomic Fields, Springer-Verlag, New York, (1982).

[4] Diamond, J.: The p-adic Gamma Measures, Proc. Amer. Math. Soc., 75(2), 211-217, (1979).

[5] Diamond, J.: The p-adic Log Gamma Function and p-adic Euler Constants, Trans. Amer. Math. Soc., 233, 321-337, (1977).

[6] Lang, S.: Introduction to Modular Forms, Springer-Verlag, Berlin Heidelberg, (1976).

[7] Katz, N.: p-Adic Properties of Modular Schemes and Modular Forms, In: Kuijk W., Serre JP. (eds) Modular Functions of One Variable III, Lecture Notes in Mathematics, vol 350. Springer, Berlin, Heidelberg, (1973).

[8] Katz, N.: p-adic L-functions via Moduli of Elliptic Curves, Proc. 1974 AMS Arcata Summer Institute in Algebraic Geometry, PSPM 29, AMS, Providence (1975).

[9] Koblitz, N.: p-adic Numbers, p-adic Analysis and Zeta-Functions, Springer-Verlag, New York, (1984).

[10] Koblitz, N.: p-adic Analysis: a Short Course on Recent Work, London Mathematical Society Lecture Note 46, Cambridge University Press, Cambridge, New York, (1980).

\section{Affiliations}

\section{ALTAN ERDOĞAN}

AdDress: Gebze Technical University, Department of Mathematics, 41400, Gebze, Turkey

E-MAIL: alerdogan@gtu.edu.tr

ORCID ID: 0000-0001-5113-1906 